\title{
Inhibition of GP130/STAT3 and EMT by combined bazedoxifene and paclitaxel treatment in ovarian cancer
}

\author{
SUN-AE PARK, LEE KYUNG KIM, HYE MIN PARK, HEE JUNG KIM and TAE-HWE HEO \\ Laboratory of Pharmacoimmunology, Integrated Research Institute of Pharmaceutical Sciences and \\ BK21 FOUR Team for Advanced Program for Smart Pharma Leaders, College of Pharmacy, \\ The Catholic University of Korea, Bucheon-si, Gyeonggi-do 14662, Republic of Korea
}

Received September 10, 2021; Accepted December 2, 2021

DOI: $10.3892 /$ or.2022.8263

\begin{abstract}
The interleukin 6 (IL-6)/glycoprotein 130 (GP130)/signal transducer and activator of transcription 3 (STAT3) signalling pathway, with GP130 as an intermediate membrane receptor, is involved in the survival, metastasis, and resistance of ovarian cancer. Bazedoxifene, an FDA-approved drug, is an inhibitor of GP130 and a selective estrogen modulator (SERM). We studied the mechanism of the combination therapy of bazedoxifene and paclitaxel in inhibiting the IL-6-mediated GP130/STAT3 signaling pathway in ovarian cancer. Surface plasmon resonance (SPR) was used to assess the binding of bazedoxifene to GP130. Migration, invasion, and apoptosis of ovarian cancer cells were assessed using bazedoxifene and paclitaxel. In addition, we determined the effects of bazedoxifene and paclitaxel alone or in combination on the GP130/STAT3 pathway and epithelial-mesenchymal transition (EMT). The results revealed that the combination of bazedoxifene and paclitaxel suppressed cell viability, migration, and invasion in the ovarian cancer cells. In addition, the combination treatment increased apoptosis. Furthermore,
\end{abstract}

Correspondence to: Mrs. Hee Jung Kim or Professor Tae-Hwe Heo, Laboratory of Pharmacoimmunology, Integrated Research Institute of Pharmaceutical Sciences and BK21 FOUR Team for Advanced Program for Smart Pharma Leaders, College of Pharmacy, The Catholic University of Korea, 43 Jibong-ro, Bucheon-si, Gyeonggi-do 14662, Republic of Korea

E-mail: hjk0114@catholic.ac.kr

E-mail: thhur92@catholic.ac.kr

Abbreviations: IL-6, interleukin-6; STAT3, signal transducer and activator of transcription 3; GP130, glycoprotein 130; EMT, epithelial-mesenchymal transition; SPR, surface plasmon resonance; ECACC, European Collection of Cell Culture; KCLB, Korean Cell Line Bank; ATCC, American Type Culture Collection; CCK-8, Cell Counting Kit-8; RIPA, radioimmunoprecipitation assay; NRF, National Research Foundation of Korea

Key words: ovarian cancer, signalling pathway, interleukin-6, signal transducer and activator of transcription 3, epithelial-mesenchymal transition, bazedoxifene, paclitaxel bazedoxifene combined with paclitaxel inhibited the growth of ovarian cancer cells in a xenograft tumour model. This combination reduced STAT3 phosphorylation and suppressed gene expression and EMT. In conclusion, inhibition of GP130/STAT3 signalling and EMT via a combination of bazedoxifene and paclitaxel could be used as a therapeutic strategy by which to overcome ovarian cancer.

\section{Introduction}

Ovarian cancer is the seventh most common cancer in women, and its incidence is the highest among all types of cancer in women worldwide. Ovarian cancer is the most lethal gynaecological cancer, with a 5-year survival rate of only $50 \%$ (1). Until recently, there has been slow progress in the development of treatment strategies for this deadly disease. A combination of surgery and chemotherapy is used as a standard therapy for patients with ovarian cancer. Paclitaxel, a widely used first-line chemotherapeutic agent against ovarian cancer, causes apoptosis of tumour cells by arresting the G2/M phase in the cell cycle, which is important for cell division (2). However, several clinical studies have reported the occurrence of resistance to and toxicity of paclitaxel, with limited results in the treatment of ovarian cancer $(3,4)$. Therefore, there is a need to develop low-toxicity formulations with distinct molecular targets to improve ovarian cancer treatment outcomes.

Inflammatory cytokines have been shown to play several roles in cancerous tumour growth in the ovaries and in the production of pre-inflammatory cytokines. Interleukin-6 (IL-6) is a major immune-regulatory cytokine that has been implicated in ovarian cancer growth $(5,6)$. Glycoprotein 130 (GP130) is a co-receptor subunit of the IL-6 cytokine family that contains IL-6, IL-11, IL-27, oncostatin M (OSM), leukaemia inhibitory factor (LIF), ciliary neurotrophic factor (CNTF), cardiotrophin 1 (CT-1), and cardiotrophin-like cytokine factor (CLCF1). GP130 mediates a wide variety of biological processes and plays an important role in resistance to apoptosis, and promotion of angiogenesis, proliferation, metastasis, and tumourigenesis (7-11). Thus, inhibition of GP130 is a promising strategy for cancer treatment.

The IL-6/GP130/signal transducer and activator of transcription 3 (STAT3) pathway is involved in a variety of oncogenic processes (12-15). In addition, constitutive 
activation of STAT3 is maintained in ovarian cancer and plays an important role in cancer cell growth, cell cycle progression, and invasion $(16,17)$. GP130 acts as a signal transducer for this signalling axis, which is closely related to cancer progression. However, the use of direct GP130 antagonists is extremely limited. A recent study found that bazedoxifene, an FDA-approved drug, targets the GP130 D1 domain and interferes with the IL-6/GP130 interaction. Thus, inactivating the dimerisation of the IL-6/IL-6R/GP130 heterotrimer can inhibit downstream STAT3 phosphorylation (18). Recently, it has been reported that bazedoxifene exhibits anticancer activity by inhibiting STAT3 signalling in cervical cancer, breast cancer, pancreatic cancer, and colon cancer (19-23). Research on bazedoxifene is expected to accelerate as well as clinical treatment with bazedoxifene on a variety of cancers dependent on IL-6/GP130/STAT3. However, the potential therapeutic effects in regards to ovarian cancer have not yet been investigated.

In the present study, combination therapy with bazedoxifene and paclitaxel was found to inhibit the IL-6-mediated GP130/STAT3 signalling pathway, induced apoptosis in ovarian cancer cells, inhibited epithelial-mesenchymal transition (EMT), and inhibited tumour growth in human ovarian cancer xenografts. Targeting the GP130/STAT3 signalling pathway could act as a new therapeutic strategy for ovarian cancer.

\section{Materials and methods}

Surface plasmon resonance (SPR) analysis. SPR analysis was performed using the BIAcore T200 model (GE Healthcare) at room temperature with PBSTT buffer (1X PBS, $0.05 \%$ Triton X-100, 0.05\% Tween-20) containing 5\% dimethyl sulfoxide (DMSO; Sigma-Aldrich; Merck KGaA). The pH scouting for GP130 (Sino Biological, China, or ANRT, Korea) and IL-6R $\alpha$ (PeproTech) immobilisation was performed in $10 \mathrm{mM}$ acetate buffer at $\mathrm{pH} 4.0,4.5,5.0$, and 5.5. GP130 and IL-6R $\alpha$ were immobilised on a CM5 chip to 2,000 and 1,000 response units (RU) with standard amine coupling at $\mathrm{pH}$ 4.5. Bazedoxifene was injected into the GP130 and IL-6R $\alpha$-immobilised flow cell at concentrations of $50,100,125,150,175$, and $200 \mu \mathrm{M}$ with a flow rate of $20 \mu \mathrm{l} / \mathrm{min}$ for $250 \mathrm{sec}$ and allowed to dissociate for $600 \mathrm{sec}$. The T-200 BIAevaluation software v3.1 (GE Healthcare) was used to subtract the references and determine steady-state $\mathrm{K}_{\mathrm{D}}$. Between the sample series, a solvent correction cycle was run to adjust for referencing errors caused by refractive index mismatches between the running buffer and samples (24).

Bioassay with IL-6-dependent DS-1 cells. The DS-1 cell line was obtained from the American Type Culture Collection (ATCC). DS-1 cells were cultured in RPMI-1640 medium (Welgene) containing 10\% FBS (HyClone; Thermo Fisher Scientific, Inc.), $1 \%$ penicillin-streptomycin (Corning, Inc.), $1 \mathrm{ng} / \mathrm{ml}$ recombinant human IL-6 (BioLegend). After starvation of the DS- 1 cells without IL- 6 for $24 \mathrm{~h}$, various concentrations of IL-6 $(0,0.01,0.05,0.1,0.5,1,5$, and $10 \mathrm{ng} / \mathrm{ml})$ were added to $5 \times 0^{4}$ cells/well and incubated for $72 \mathrm{~h}$. The D-Plus ${ }^{\mathrm{TM}}$ Cell Viability Assay Kit (DonginBiotech) was used to assess the cell proliferation. Next, $10 \mu \mathrm{l}$ of the kit reagent was added to each well and incubated for $4 \mathrm{~h}$ in a $\mathrm{CO}_{2}$ incubator. After incubation, the optical density (OD) was measured at $450 \mathrm{~nm}$.

IL-6 inhibitory bioassay. After starvation of DS-1 cells without IL-6 for $24 \mathrm{~h}$, various concentrations of bazedoxifene (1, 2.5, 5, 7.5, 10, 12.5, and $15 \mu \mathrm{M}$ ) (Sigma-Aldrich; Sigma-Aldrich; Merck KGaA) in the presence of IL-6 (10 ng/ml) were added to $5 \times 10^{4}$ cells/well and incubated for $72 \mathrm{~h}$. The D-Plus ${ }^{\mathrm{TM}}$ Cell Viability Assay Kit (DonginBiotech) was used to measure cell proliferation. Next, $10 \mu \mathrm{l}$ of the kit reagent was added to each well and incubated for $4 \mathrm{~h}$ in a $\mathrm{CO}_{2}$ incubator. After incubation, the OD was measured at $450 \mathrm{~nm}$ wavelenth.

Transfection of small interfering RNA (siRNA). siRNAs against negative control siRNA (siNC) and STAT3 (siSTAT3) were purchased from Genolution (Genolution Pharmaceutical Inc.). Cells were seeded in 96-well plates at a density of $1 \times 10^{4}$ cells/well. Cells were transfected with $30 \mathrm{nM}$ siRNA in phosphate-buffered saline (1X PBS); a G-Fectin Kit (Genolution Pharmaceutical Inc.) was used according to the manufacturer's instructions. siRNA-transfected cells were used in the in vitro assays $48 \mathrm{~h}$ after transfection. The target sequences of siNC and siSTAT3 are listed in Table SI. The efficiency of siRNA-based STAT3 knockdown transfection was assessed by western blotting and then the cells were used for subsequent experiments.

MTT cell viability assay in ovarian cancer cell lines. The A2780 cell line was purchased from the European Collection of Cell Cultures (ECACC, London, UK). The OVCA433 cell line was provided by the Korea Gynecologic Cancer Bank through the Bio and Medical Technology Development Program of the Ministry of Science, Information and Communication Technology, and Future Planning (MSIP). SKOV3 and TOV112D cell lines were purchased from the Korean Cell Line Bank (KCLB) and American Type Culture Collection (ATCC). The subtypes of the ovarian cancer cell lines were as follows: A2780, not specified; OVCA433, serous; SKOV3, serous; and TOV112D, endometrioid (25). Ovarian cancer cell lines were seeded in 96-well plates at a density of 5,000 cells/well. The next day, bazedoxifene $(1,2,4,6,8$, and $10 \mu \mathrm{M})$, paclitaxel $(0.005,0.01$, and $0.05 \mu \mathrm{g} / \mathrm{ml})$ (Bristol-Myers Squibb), and a combination of bazedoxifene and paclitaxel was added in triplicate to the plates for $48 \mathrm{~h}$. The cells were incubated at $37^{\circ} \mathrm{C}$. Then, $100 \mu \mathrm{l}$ of N,N-dimethylformamide (Sigma-Aldrich; Merck $\mathrm{KGaA}$ ) solubilisation solution was added to each well and incubated for $2 \mathrm{~h}$. After $2 \mathrm{~h}$, the solution was removed by suction, and the cells stained with DMSO were lysed. The OD was measured at $450 \mathrm{~nm}$ wavelength. The half-maximal inhibitory concentration $\left(\mathrm{IC}_{50}\right)$ was analysed using the GraphPad Prism software (version 7.0; GraphPad Software, Inc.). The combination index (CI) was calculated using CompuSyn software ver 1.0 (ComboSyn, Inc.). The CI values indicate an antagonistic effect when $>1$, an additive effect when equal to 1 , and a synergistic effect when $<1$ (23).

Clonogenic formation assay. Each ovarian cancer cell line was seeded at approximately 50,000 cells in a 60-mm dish. After $6 \mathrm{~h}$, bazedoxifene and paclitaxel, and the combination of bazedoxifene and paclitaxel were added to the cell medium 
at each concentration (B: $10 \mu \mathrm{M}, \mathrm{P}: 0.05 \mu \mathrm{g} / \mathrm{ml}$, OVCA433; B: $6 \mu \mathrm{M}, \mathrm{P}: 0.005 \mu \mathrm{g} / \mathrm{ml}$, SKOV3; B: $6 \mu \mathrm{M}, \mathrm{P}: 0.01 \mu \mathrm{g} / \mathrm{ml}$, A2780; B: $6 \mu \mathrm{M}$, P: $0.01 \mu \mathrm{g} / \mathrm{ml}$, TOV112D). Cells that formed colonies within 1 to 3 weeks were fixed with glutaraldehyde $(6.0 \% \mathrm{v} / \mathrm{v})$, stained with crystal violet $(0.5 \% \mathrm{w} / \mathrm{v})$, and photographed using a ChemiDoc imaging system (Bio-Rad Laboratories). The intensities of the images were quantified using ImageJ software 1.5 a (National Institutes of Health).

Wound-healing assay and Matrigel invasion assay. Ovarian cancer cell lines were grown to approximately $90 \%$ in each 6-well plate. After starvation in serum-free medium for $24 \mathrm{~h}$, a wound-healing assay was performed in the same medium. A single layer of cells was scraped to form an artificially homogeneous wound. Afterwards, each concentration of the drug (B: $10 \mu \mathrm{M}, \mathrm{P}: 0.05 \mu \mathrm{g} / \mathrm{ml}$, OVCA433; B: $6 \mu \mathrm{M}, \mathrm{P}: 0.005 \mu \mathrm{g} / \mathrm{ml}$, SKOV3; B: $6 \mu \mathrm{M}, \mathrm{P}: 0.01 \mu \mathrm{g} / \mathrm{ml}, \mathrm{A} 2780$; B: $6 \mu \mathrm{M}, \mathrm{P}: 0.01 \mu \mathrm{g} / \mathrm{ml}$, TOV112D) was administered, and images were captured under a microscope [Primo Vert (Carl Zeiss Inc.; x100 magnification] at 0,24 , and $48 \mathrm{~h}$, and the degree of cell migration was measured using ImageJ software. All experiments were conducted independently in triplicates (19).

Matrigel invasion assays were performed using a BD BioCoat Matrigel Invasion Chamber (BD Bioscience). Ovarian cancer cell lines $\left(5 \times 10^{4}\right.$ cells) were seeded inside the chamber in serum-free medium containing $300 \mu \mathrm{l}$ of drugs. Five hundred microlitres of the medium containing serum was placed outside the chamber. After $48 \mathrm{~h}$ of incubation, the cells that infiltrated the chamber were stained using the Differential Quik Stain Kit (Diff Quik, Sysmex). Images were captured with a microscope [Primo Vert (Carl Zeiss Inc.; x100 magnification], and the number of cells invading the chamber was measured using ImageJ software. All experiments were independently conducted in triplicate.

Western blotting. Cells were lysed in $100 \mu \mathrm{l}$ of radioimmunoprecipitation assay (RIPA) buffer (Thermo Fisher Scientific Inc.) and centrifuged at $13,000 \mathrm{x}$ g for $15 \mathrm{~min}$ at $4^{\circ} \mathrm{C}$. Protein concentration was measured using the Pierce BCA Protein Assay Kit (Thermo Fisher Scientific, Inc.). Proteins were quantified to $30 \mathrm{ng}$ and boiled in $5 \mathrm{X}$ sample buffer, separated on a $10 \%$ SDS-polyacrylamide gel, and transferred to polyvinylidene difluoride (PVDF) membranes (Millipore) by electrophoresis. The membranes were blocked for $1 \mathrm{~h}$ at room temperature with $3 \%$ BSA in $1 \mathrm{X}$ Tris-buffered saline containing 0.1\% Tween 20 (Sigma-Aldrich; Merck KGaA) and anti- $\beta$-actin (cat. no. 8457S; dilution 1:1,000), anti-estrogen receptor $\alpha(\mathrm{ER} \alpha)$ (cat. no. 13258S; 1:500), anti-Mcl-1 (cat. no. 5354S; 1:1,000), anti-Bcl-xl (cat. no. 2764S; 1:500), anti-Bcl-2 (cat. no. 3498S; 1:500), anti-Bax (cat. no. 2772S; 1:500), anti-E-cadherin (cat. no. 3195S; 1:500), anti-N-cadherin (cat. no. 4061S; 1:500), anti- $\beta$-catenin (cat.no. 9562S; $1: 1,000$ ), anti-vimentin (cat. no. 5741S; $1: 1,000$ ), anti-Snail (cat. no. 3879S; 1:500), anti-Twist (cat. no. 69366S; 1:500), anti-Claudin-1 (cat. no. 4933S; 1:500), anti-phospho-STAT3 (Tyr705) (cat. no. 9131S; 1:500), anti-STAT3 (cat. no. 12640S; 1:500), anti-phospho-ERK 1/2 (cat. no. 4695S; 1:1,000), anti-ERK 1/2 (cat. no. 9101S; 1:1,000), anti-phospho-p38 (cat. no. 4511S; 1:1,000), anti-p38 (cat. no. 3690S; 1:1,000), anti-phospho-Akt (cat. no. 4060L; 1:1,000), anti-Akt (cat. no. 2920S; 1:1,000), anti-cyclin D1 (cat. no. 2978T; 1:1,000), anti-CDK4 (cat. no. 12790T; 1:1,000), anti-CDK6 (cat. no. 3136T; 1:1,000), anti-p21 ${ }^{\text {waf1 }}$ (cat. no. 2947T; 1:1,000), anti-p27 ${ }^{\mathrm{kipl}}$ (cat. no. 3686T; 1:1,000), anti-PARP (cat. no. 9542S; 1:1,000), and anti-cleaved caspase-3 (cat. no. 9661S; 1:1,000) (all obtained from Cell Signalling Technologies, Inc.), anti-estrogen receptor $\beta(\mathrm{ER} \beta)$ (cat. no. ab3576; 1:1,000) (Abcam, Inc,), and anti-phospho-GP130 (cat. no. SC-377572; 1:500), and anti-GP130 (cat. no. SC-376280; 1:500) (Santa Cruz Biotechnology, Inc.) antibody was stored at $4^{\circ} \mathrm{C}$ overnight. An anti- $\beta$-actin antibody was used as an internal control. Membranes were washed with $1 \mathrm{X}$ Tris-buffered saline containing $0.1 \%$ Tween-20 (TBST) and incubated with AffiniPure goat anti-rabbit IgG secondary antibody (Jackson Immunoresearch) and goat anti-mouse IgG secondary antibody (Bethyl Laboratories) for $2 \mathrm{~h}$ at room temperature. After washing again with TBST, an enhanced chemiluminescence (ECL) kit (Thermo Fisher Scientific, Inc.) was used to detect the signal, and the intensity was quantified using ImageJ software. Data were obtained from at least three biological replicates (23).

Flow cytometric analysis of apoptosis. For cell death analysis, after treatment with bazedoxifene, paclitaxel, or their combination for $48 \mathrm{~h}$ in ovarian cancer cell lines, the level of apoptosis was quantified by flow cytometry. Experiments were conducted using the Annexin V-FITC Apoptosis Detection Kit (BD Pharmingen), and apoptosis was analysed by flow cytometry. Cells were sorted using a FACSCanto II (BD Biosciences), and apoptotic cells were analysed using BD FACS Diva software version 6.2 (BD Biosciences). The analysis was performed in triplicate (20).

Tumour xenografts in mice. A total of 24 female BALB/c nude mice (5 weeks of age, body weight 18-20 g, Orient Bio) were maintained under aseptic conditions with constant at $25^{\circ} \mathrm{C}$ temperature and $50 \%$ humidity with a $12 / 12$-h light/dark cycle. (Catholic University protocol). Water and sterile food were provided daily at the Catholic University. The animals had access to water and food ad libitum. Animal experiments were approved by the Institutional Animal Care and Use Committee (IACUC) of the Catholic University of Korea (approval no. CUK-IACUC-2019-026-01). All experimental work complied with the legal obligations and federal guidelines for the care and maintenance of laboratory animals.

Each mouse received a subcutaneous injection of $1 \times 10^{7}$ OVCA433 cells in $100 \mu \mathrm{l}$ PBS into the dorsal scapula area, and the tumour volumes continued to be monitored. The tumour size was measured every 3 days by using calipers, while the bodyweight of the mice was measured. The tumours developed 10 days after implantation, and the tumour size reached approximately $200 \mathrm{~mm}^{3}$. A total of 24 mice were randomly assigned into four groups ( $n=6$ per group). Next, the mice were randomly divided into vehicle control, bazedoxifene, paclitaxel, and combination groups. Bazedoxifene ( $4 \mathrm{mg} / \mathrm{kg}$ ) was administered by gavage five times a week, and paclitaxel $(10 \mathrm{mg} / \mathrm{kg})$ was administered by intraperitoneal injection twice weekly $(19,26)$. Calipers were used to measure the tumour size once every 2 days. Tumour volume was calculated using a simplified equation to estimate the rotational 

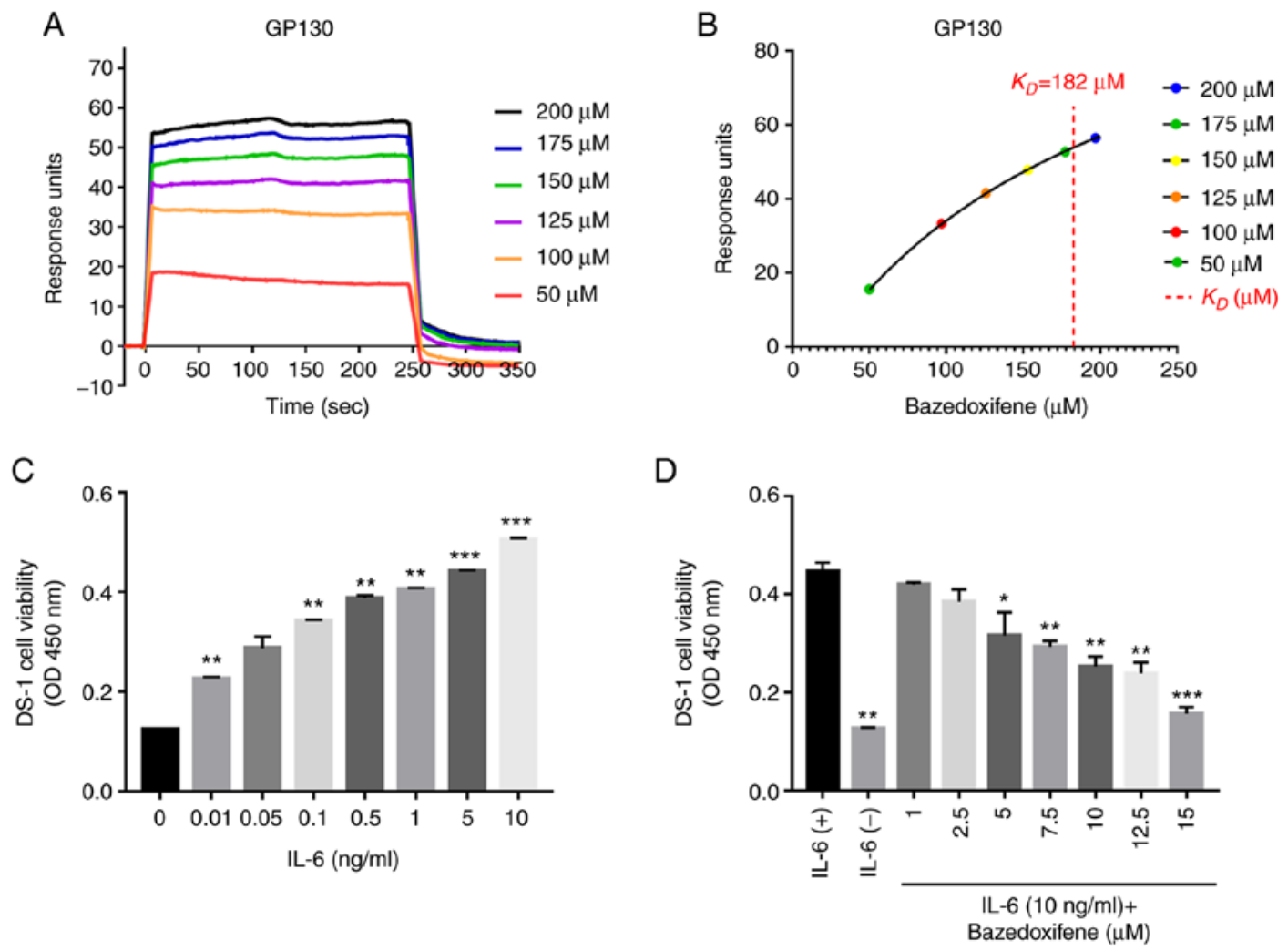

Figure 1. Characterisation of bazedoxifene and anti-IL-6 activity in vitro. (A) For surface plasmon resonance (SPR) analysis, bazedoxifene binding to GP130 was immobilised on a CM5 sensor chip and bazedoxifene was injected into the flow cells. (B) T-200 BIA evaluation software was used to subtract the references and determine the steady-state $K_{\mathrm{D}}$. (C) The IL-6-dependent proliferation of DS-1 cells. DS-1 cells were incubated with various concentrations of IL-6 for $72 \mathrm{~h}$ for the CCK-8 assay; $10 \mathrm{ng} / \mathrm{ml}$ IL- 6 was determined as the optimal concentration for subsequent IL-6 inhibitory assays. (D) Inhibition of IL-6-induced proliferation of DS-1 cells by bazedoxifene. DS-1 cells were incubated with IL-6 $(10 \mathrm{ng} / \mathrm{ml})$ in the presence of different concentrations of bazedoxifene $\left({ }^{*} \mathrm{P}<0.05,{ }^{* *} \mathrm{P}<0.01,{ }^{* * *} \mathrm{P}<0.001\right)$. IL-6, interleukin 6.

ellipsoid (length $\mathrm{x}$ width ${ }^{2} \mathrm{x} 0.5$ ). At the end of the experiment, the mice were then sacrificed by $\mathrm{CO}_{2}$ inhalation $(50 \%$ of the chamber volume/min). Whenever the gradual displacement method is used, the $\mathrm{CO}_{2}$ flow must be maintained for at least 1 min after respiratory arrest. Each tumour was harvested 16 days post-treatment and measured, and the maximum tumor diameter did not exceed $2 \mathrm{~cm}$.

Hematoxylin and eosin $(H \& E)$ staining. The mice were sacrificed 16 days after the drug administration. Tumour tissues were collected, fixed in $4 \%$ paraformaldehyde for $24 \mathrm{~h}$, washed in 1X PBS, and embedded in paraffin. Sections $(2-\mu \mathrm{m})$ were stained with $\mathrm{H} \& \mathrm{E}$ following standard procedures. After staining, dehydration was performed. After fixing with mineral oil, cover slipping was performed.

Data analysis. Results are presented as mean \pm standard deviation (SD). Data were assessed using Excel and GraphPad Prism software (version 7.0; GraphPad Software, Inc.). Two-way ANOVA followed by Bonferroni's post hoc test (multiple comparisons) were performed with GraphPad Prism 7.0. $\mathrm{P}<0.05$ was considered to indicate a statistically significant difference.

\section{Results}

Characterisation of bazedoxifene and anti-IL-6 activity in vitro. Bazedoxifene targets GP130, and in the present study, surface plasmon resonance (SPR) analysis was performed to monitor its binding affinity to GP130. Recombinant GP130 and rIL-6R $\alpha$ were covalently cross-linked to the dextran matrix of the CM5 chip, and various concentrations of bazedoxifene were passed through the surface and the reference surface. To investigate the association between GP130 binding affinity and anticancer activity, response units (RU) for each concentration of bazedoxifene were detected by SPR analysis (Fig. 1A). An established SPR-based biosensor assay was used to investigate the ability of bazedoxifene to bind GP130. A single binding site model was fitted to the data to calculate the $\mathrm{K}_{\mathrm{D}}$ value of bazedoxifene at $182.7 \mu \mathrm{M}$ (Fig. 1B). Solvent-corrected binding curve fit was analysed using the Biacore evaluation software. The IL-6R $\alpha$ data are shown in Fig. S1. Interestingly, bazedoxifene has a higher binding affinity for GP130. The IL-6-dependent DS-1 cell line was confirmed to be dependent on IL- 6 , and the optimal IL-6 concentration for inhibition analysis was determined to be $10 \mathrm{ng} / \mathrm{ml}$ (Fig. 1C). To confirm whether bazedoxifene inhibited cell proliferation by blocking the binding of IL-6/GP130, different concentrations of bazedoxifene were added to DS-1 cells treated with IL-6 $(10 \mathrm{ng} / \mathrm{ml})$. The proliferation of cells was inhibited by bazedoxifene in a concentration-dependent manner (Fig. 1D). The results showed that bazedoxifene inhibited cell proliferation by inhibiting the binding of IL-6 to GP130.

Bazedoxifene, paclitaxel and their combination inhibit cell viability and proliferation of ovarian cancer cells. It was 

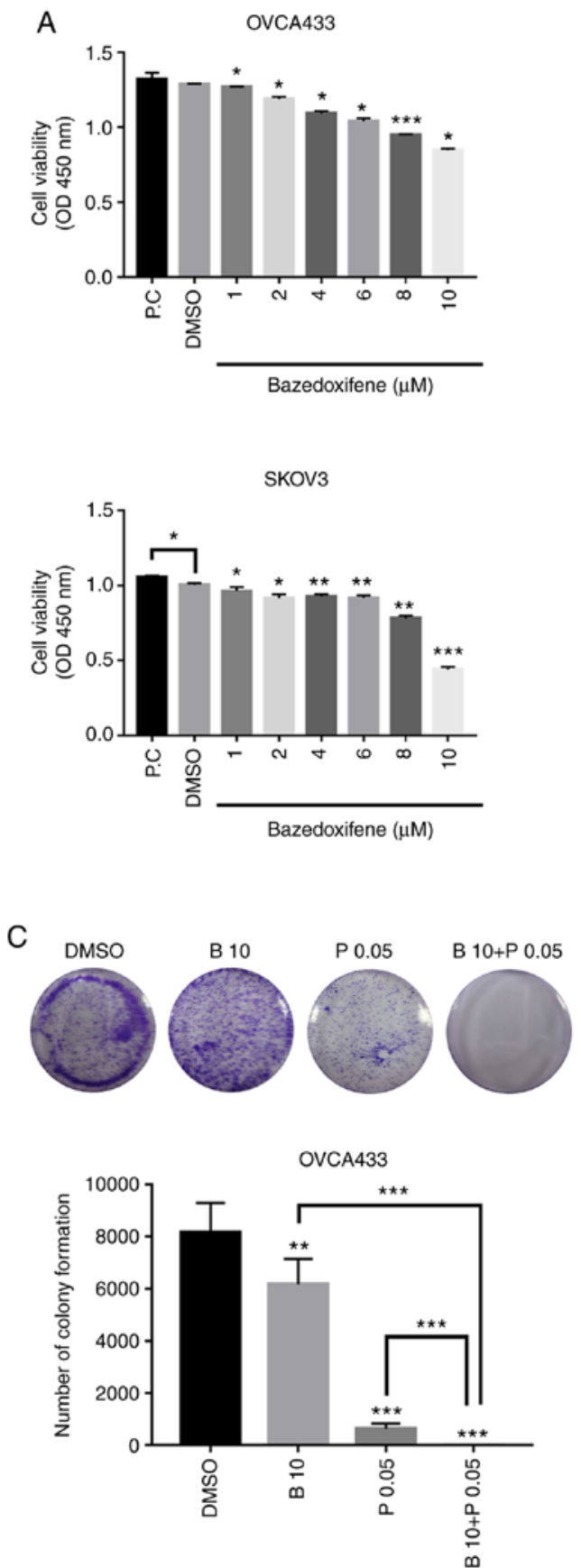

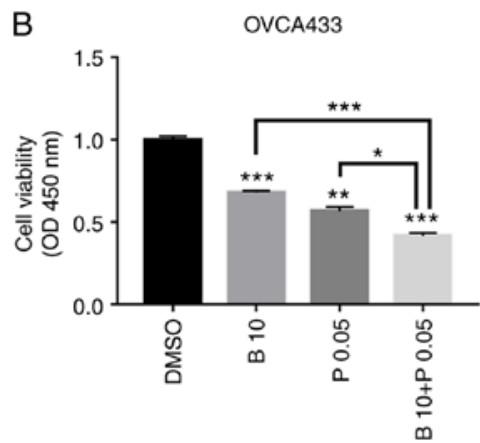

$\mathrm{Cl}: 0.54636$

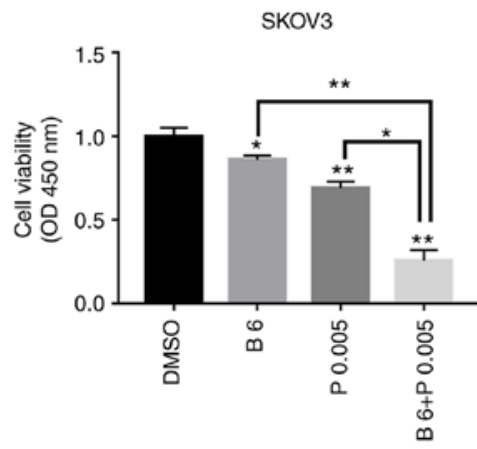

$\mathrm{Cl}: 0.43954$

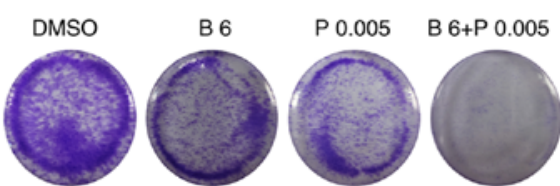

SKOV3

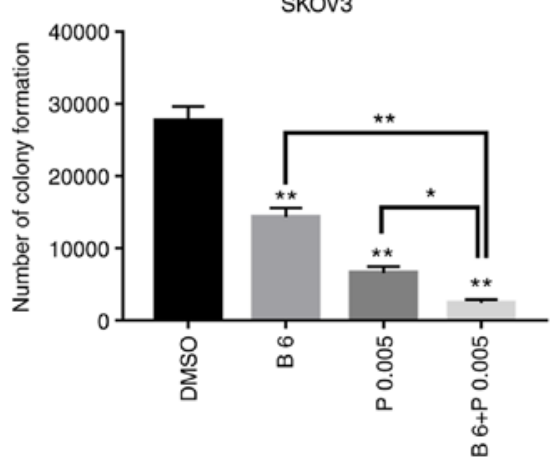

B: Bazedoxifene $(\mu \mathrm{M})$, P: Paclitaxel $(\mu \mathrm{g} / \mathrm{ml})$

Figure 2. Bazedoxifene, paclitaxel and their combination inhibit cell viability and proliferation of ovarian cancer cells. (A) Ovarian cancer OVCA433 and SKOV3 cells were treated with bazedoxifene at different concentrations in triplicate for $48 \mathrm{~h}$ and processed for MTT assay to analyse cell viability ("P $<0.05$, $\left.{ }^{* *} \mathrm{P}<0.01,{ }^{* * *} \mathrm{P}<0.001\right)$. (B) Ovarian cancer OVCA433 and SKOV3 cells were treated with bazedoxifene (B), paclitaxel $(\mathrm{P})$ and their combination $(\mathrm{B}+\mathrm{P})$ at the indicated concentrations in triplicate for $48 \mathrm{~h}$ and processed for MTT assay to analyse cell viability $\left({ }^{*} \mathrm{P}<0.05,{ }^{* *} \mathrm{P}<0.01,{ }^{* * * *} \mathrm{P}<0.001\right)$. (C) Colony formation assay revealed that the combination of bazedoxifene and paclitaxel $(\mathrm{B}+\mathrm{P})$ significantly reduced the colony growth of ovarian cancer OVCA433 and SKOV3 cells. Data are representative of results obtained from three independent proliferation assays and three replicates were analysed in each assay $\left({ }^{*} \mathrm{P}<0.05,{ }^{* * *} \mathrm{P}<0.01\right.$, $\left.{ }^{* * * *} \mathrm{P}<0.001\right)$. CI, combination index.

next investigated whether bazedoxifene inhibits the growth of human ovarian cancer cell lines. OVCA433, SKOV3, A2780, and TOV112D cells were treated with bazedoxifene at different concentrations in triplicate for $48 \mathrm{~h}$ and processed for MTT assay to analyse cell viability (Figs. 2A and S2A). Cell viability of OVCA433, SKOV3, A2780, and TOV112D cells were decreased by bazedoxifene in a concentration-dependent manner. To explore the synergistic inhibitory effects of bazedoxifene and paclitaxel on ovarian cancer cells, cells were treated with bazedoxifene, paclitaxel, or their combination in triplicate for $48 \mathrm{~h}$. Cell viability was further inhibited in the combined group compared to the monotherapy group, with respective $\mathrm{IC}_{50}$ values of 17.2, 8.99, 14.7 and $4.56 \mu \mathrm{M}$ (Figs. 2B and S2B). The combination index (CI) values of all combination treatments were $<1$, suggesting that combined treatment with bazedoxifene and paclitaxel and bazedoxifene-sensitized 
ovarian cancer cells were synergistic in combination treatment with paclitaxel. To confirm the anticancer ability of the drug in ovarian cancer cell lines, a clonogenic formation assay was performed (Figs. 2C and S2C). The clonogenic ability was significantly reduced in the group treated with bazedoxifene and paclitaxel alone, compared to the group treated with DMSO. In the group treated with bazedoxifene and paclitaxel, colony formation was significantly suppressed compared to that in the group treated with either drug alone. This suggests that the combination treatment of bazedoxifene and paclitaxel exerts a synergistic effect on ovarian cancer cells.

Bazedoxifene and paclitaxel or their combination inhibits migration and invasion in ovarian cancer cells. OVCA433 and SKOV3 cells were treated with bazedoxifene, paclitaxel, or their combination and allowed to migrate to the scratched area for 0,24 , and $48 \mathrm{~h}$. The yellow line indicates the width of the wound. The percentage of the migrating area in the wound-healing assay was quantified in OVCA433 and SKOV3 cells. Cell migration was significantly inhibited at $48 \mathrm{~h}$ in both bazedoxifene and paclitaxel, and bazedoxifene and paclitaxel combination treatment groups compared with the DMSO-treated control group. Among them, the group treated with bazedoxifene and paclitaxel showed more significant inhibition of cell migration (Fig. 3A). The Matrigel invasion assay was used to determine the invasion after $48 \mathrm{~h}$ in OVCA433 and SKOV3 cells treated with the combination of drugs. Compared with the control group treated with DMSO, cell invasion was significantly suppressed in both bazedoxifene and paclitaxel alone and bazedoxifene and paclitaxel combination treatment groups. In the group treated with bazedoxifene and paclitaxel, cell invasion was significantly suppressed compared to that in groups treated with bazedoxifene and paclitaxel alone (Fig. 3B). A2780 and TOV112D cells showed significant results (Fig. S3), suggesting that bazedoxifene and paclitaxel inhibited migration and invasion, respectively. However, their combination exerted more effective inhibitory effects on migration and invasion.

Bazedoxifene suppresses the expression of $p$-STAT3 and estrogen receptors in ovarian cancer cells. Bazedoxifene is a selective estrogen receptor (ER) modulator, and research on potential novel GP130 inhibitors is currently underway. We aimed to elucidate the mechanism by which bazedoxifene inhibits STAT3 signalling by targeting GP130 in ovarian cancer. As shown in Fig. 4A, SKOV3 cells were found to express ER $\alpha$, while the other cell lines tested exhibited no ER $\alpha$ expression. In addition, STAT3 phosphorylation (p-STAT3) was not observed in the A2780 and TOV112D cell lines. Accordingly, it is thought that there is a difference in the degree of drug response depending on the presence or absence of ERs. IL-6 is known to induce STAT3 phosphorylation, and we attempted to determine whether bazedoxifene can inhibit IL-6-induced STAT3 phosphorylation. A2780 and TOV112D cells that did not express phosphorylated STAT3 in serum-free medium for $24 \mathrm{~h}$ were used. In this study. It was found that IL-6 stimulated the phosphorylation of STAT3 and that bazedoxifene decreased phosphorylation in a dose-dependent manner (Fig. 4B). The mechanism by which bazedoxifene inhibits the phosphorylation of STAT3 in the ER-positive ovarian cancer cell line SKOV3 was subsequently investigated. Bazedoxifene downregulated the expression of ER $\alpha$ and p-STAT3 induced by IL-6 in SKOV3 cells. It was also confirmed that phosphorylation of STAT3 induced by IL- 6 was inhibited as the concentration of bazedoxifene increased in OVCA433 cells, which are ER-negative cells (Fig. 4C). Thus, we found that bazedoxifene inhibited STAT3 phosphorylation with and without ERs.

OVCA433 and SKOV3 cells were transfected with four different sequences of siSTAT3 (si1, si2, si3, and si4), and siSTAT3-4 showed the maximum inhibitory activity against STAT3 in the OVCA433 and SKOV3 cell lines (Fig. 4D). To confirm the ability of bazedoxifene to inhibit cell proliferation and migration and to test whether this effect is dependent on STAT3, the inhibitory effect of bazedoxifene on the proliferation and migration of OVCA433 and SKOV3 cells transfected with siSTAT3 was investigated (Figs. 4E and S4). We found that bazedoxifene had no significant effect on siSTAT3 cells. These results indicate that bazedoxifene is an inhibitory target of the STAT3 signalling pathway in ER-positive and -negative ovarian cancer cells. The whole western membrane and expression levels are shown in Fig. S5.

Combined bazedoxifene and paclitaxel inhibits IL-6-induced phosphorylation of GP130, STAT3, ERK1/2 and EMT in ovarian cancer cells. The effect of bazedoxifene binding to GP130 on STAT3 signalling, a sub-signaling pathway of GP130, was investigated. Ovarian cancer cell lines were starved for $24 \mathrm{~h}$ and treated with bazedoxifene, paclitaxel, and a combination of bazedoxifene and paclitaxel for $6 \mathrm{~h}$. Next, to evaluate the effect on sub-signaling, IL-6 $(100 \mathrm{ng} / \mathrm{ml})$ was added for $15 \mathrm{~min}$, and p-GP130, p-STAT3, p-Akt, p-p38 MAPK, p-ERK 1/2, and EMT-related antibodies were detected by western blotting (Fig. 5). Compared with the control group treated with only IL-6, the phosphorylation of GP130 (p-GP130), STAT3 (p-STAT3), ERK1/2 (p-ERK1/2), Akt (p-Akt), and p38 MAPK (p-p38-MAPK) was decreased in the bazedoxifene and paclitaxel alone group, and further decreased in the combined treatment group (Fig. 5A). The whole western membrane and expression levels are shown in Figs. S6 and S7. A2780 and TOV112D cells also showed significant results (Fig. S8A). The whole western membrane and expression levels are shown in Figs. S9 and S10. To confirm whether STAT3 signalling by drugs affected EMT, the expression of EMT markers N-cadherin, E-cadherin, $\beta$-catenin, vimentin, Snail, Twist, and Claudin-1 was assessed by western blotting. The combination treatment of bazedoxifene and paclitaxel inhibited EMT-related protein expression, with significant differences in the expression of vimentin, Snail, Twist, and Claudin-1 in OVCA433 and SKOV3 cells (Fig. 5B). The whole western membrane and expression levels are shown in Figs. S11 and S12. A2780 and TOV112D cells showed significant results (Fig. S8B). The whole western membrane and expression levels are shown in Figs. S13 and S14. These results showed that bazedoxifene and paclitaxel inhibited the signalling of GP130/STAT3 and EMT, and the combination treatment significantly inhibited this effect.

Bazedoxifene combined with paclitaxel induces growth arrest and apoptosis. It was demonstrated that bazedoxifene inhibits 
A
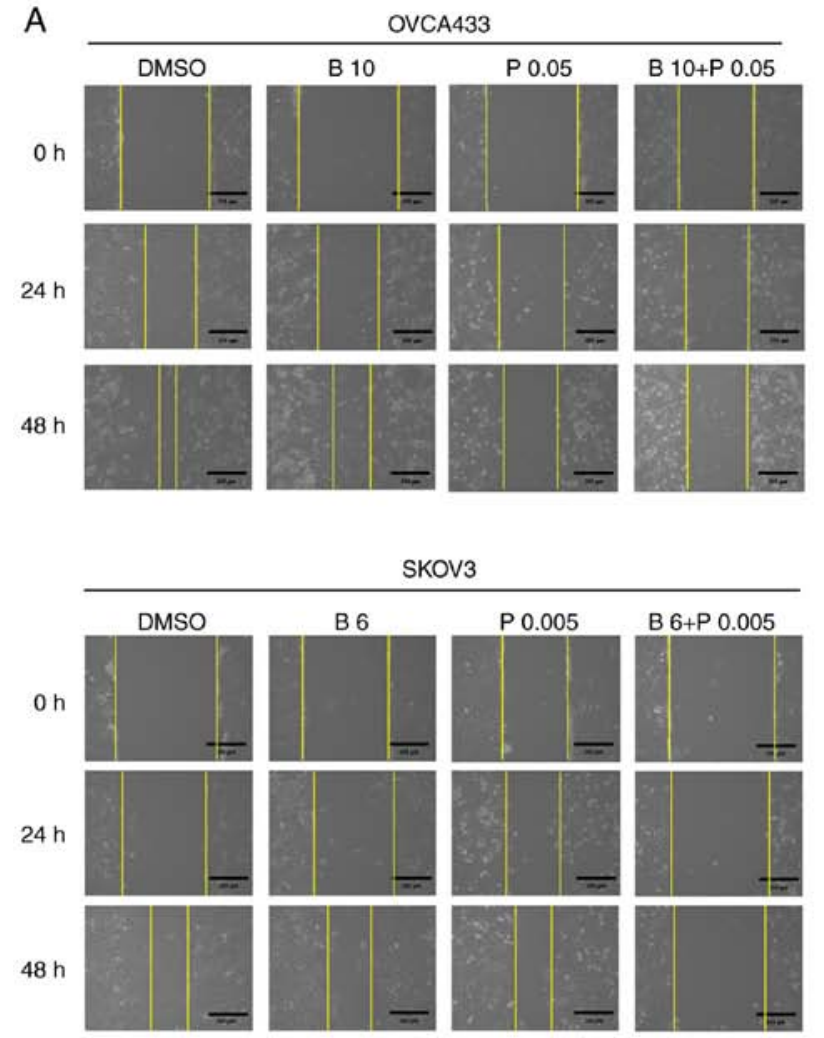

B

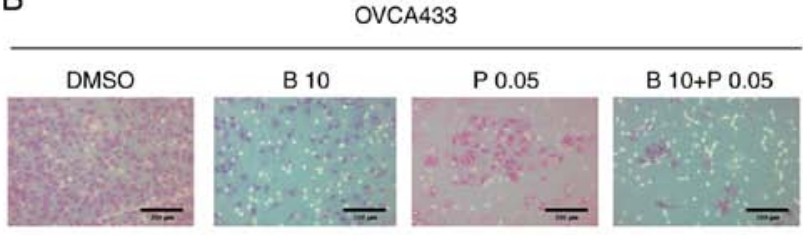

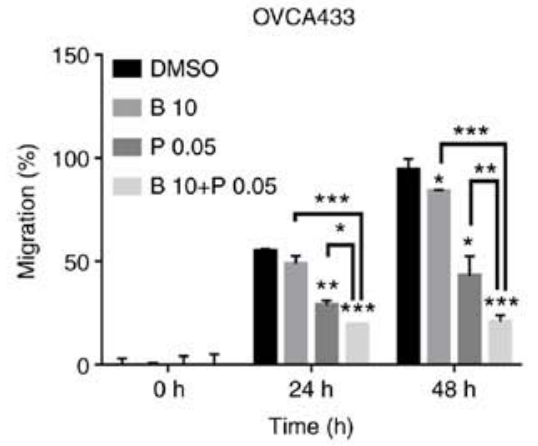

SKOV3

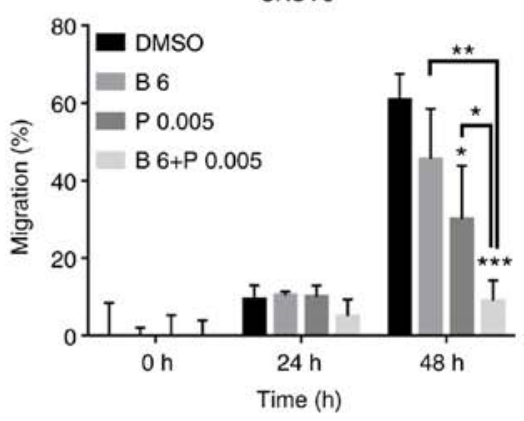

OVCA433

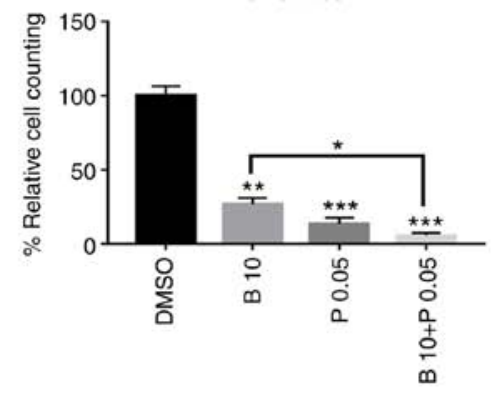

SKOV3

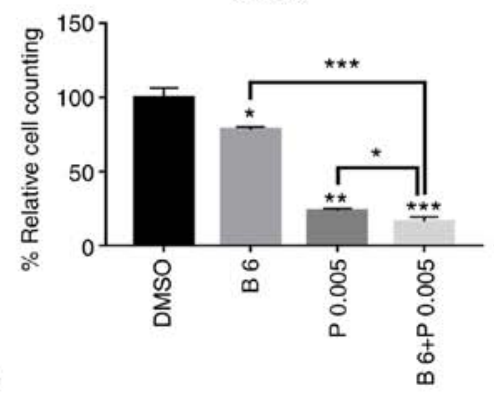

Figure 3. Bazedoxifene and paclitaxel or their combination inhibit the migration and invasion of ovarian cancer cells. (A) Ovarian cancer OVCA433 and SKOV3 cells were treated with bazedoxifene (B), paclitaxel $(\mathrm{P})$ or their combination $(\mathrm{B}+\mathrm{P})$ and allowed to migrate to the scratched area for $48 \mathrm{~h}$. Yellow coloured lines indicate a gap in the scratched area. The percentage of the migrating area in the wound-healing assay quantified in OVCA433 and SKOV3 cells is shown $\left({ }^{*} \mathrm{P}<0.05,{ }^{* *} \mathrm{P}<0.01,{ }^{* * * *} \mathrm{P}<0.001\right)$. (B) Matrigel invasion assay was used to determine the invasion after $48 \mathrm{~h}$ of treatment of OVCA433 and SKOV3 cells with a combination of bazedoxifene and paclitaxel $(\mathrm{B}+\mathrm{P})$. Bars indicate the mean \pm standard deviation of three independent experiments performed in triplicate $\left({ }^{*} \mathrm{P}<0.05,{ }^{* *} \mathrm{P}<0.01,{ }^{* * *} \mathrm{P}<0.001\right)$.

the survival, migration, and metastasis of cancer cells by inhibiting IL-6/GP130/STAT3 signalling. Furthermore, whether bazedoxifene affects the cell cycle and apoptosis in ovarian cancer cells was assessed. As observed through western blot analysis, cyclin D1, CDK4, and CDK6 were significantly decreased, and p21 and p27 were slightly increased in OVCA433 and SKOV3 cells treated with bazedoxifene and paclitaxel alone and the combination treatment. These 


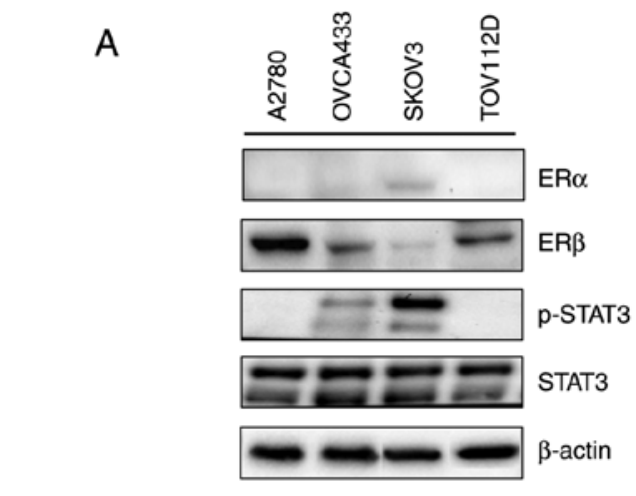

C
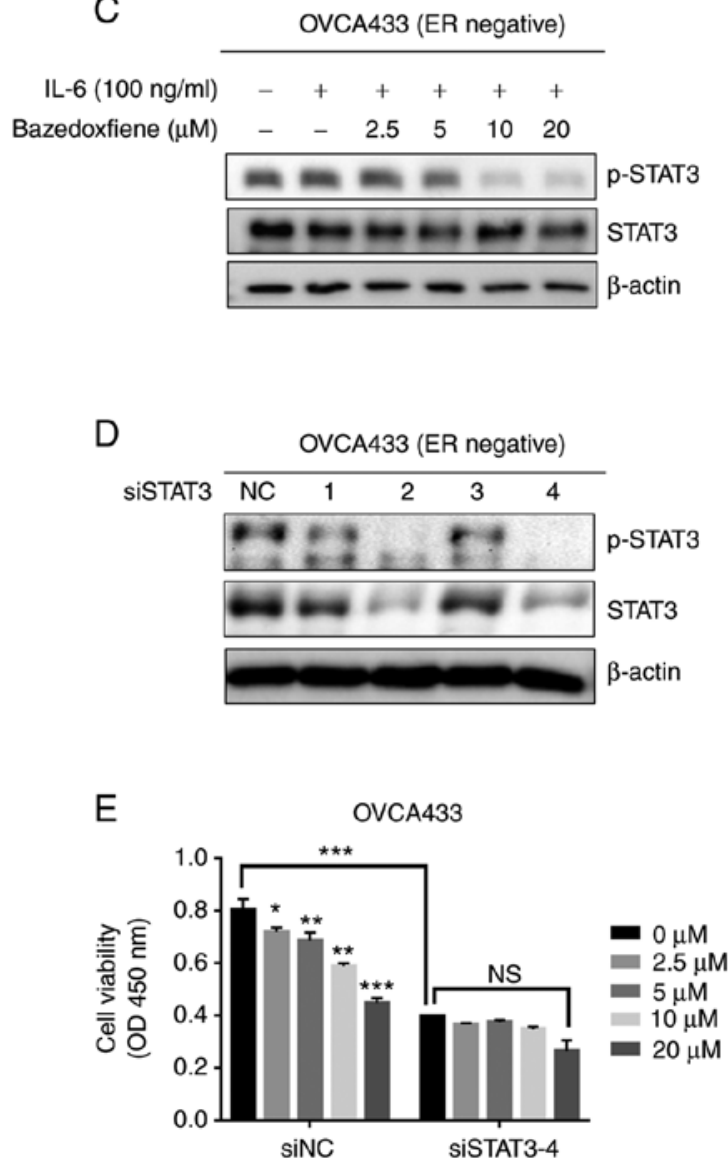

B
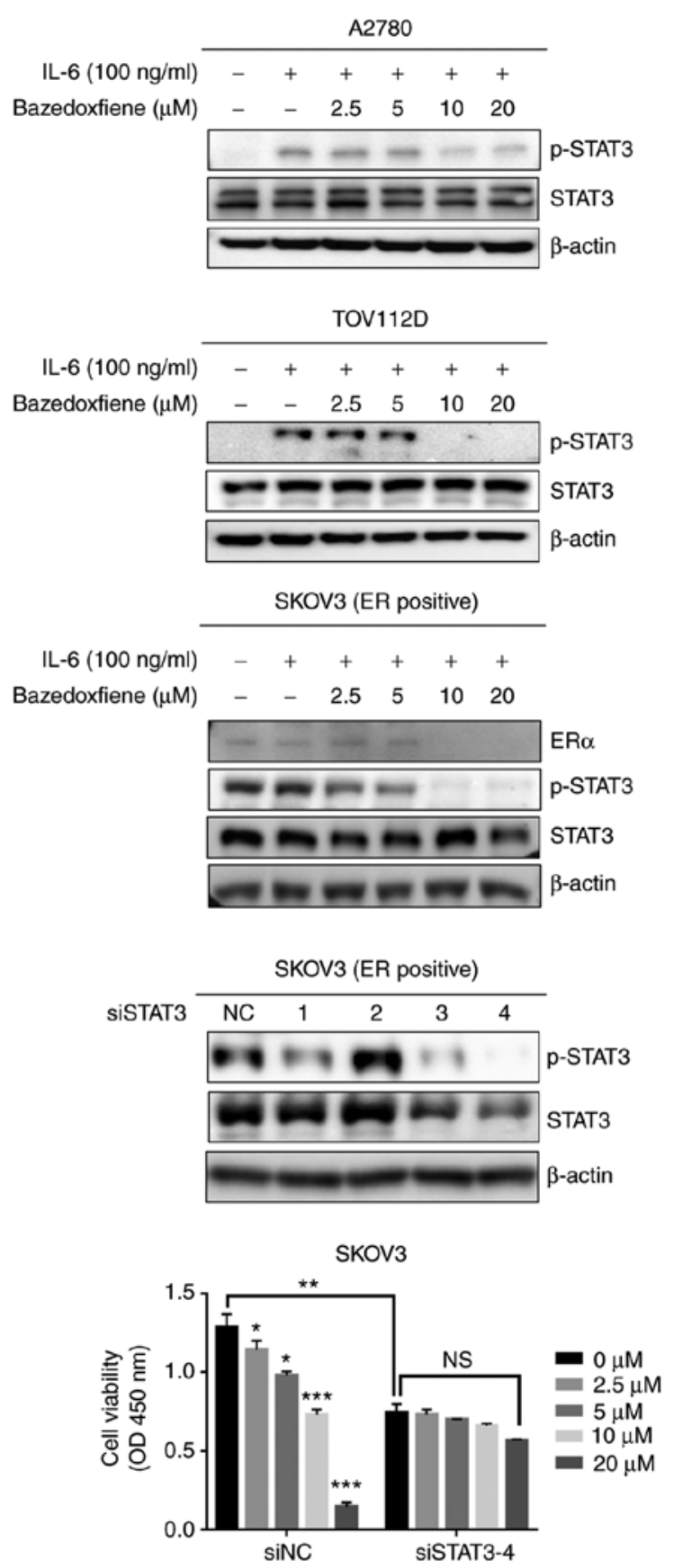

Figure 4. Bazedoxifene inhibits the expression of phospho-STAT3 and estrogen receptor in ovarian cancer cells. (A) Expression of phospho-STAT3 (p-STAT3) and estrogen receptors (ER $\alpha$ and $E R \beta$ ) was confirmed in ovarian cancer cell lines. (B) After phosphorylation was induced with IL-6 (100 ng/ml) in A2780 and TOV112D cells that did not express p-STAT3, bazedoxifene was administered at different concentrations, showing that p-STAT3 was inhibited following treatment. (C) The degree of p-STAT3 inhibition was confirmed according to the concentration of bazedoxifene in the ER-negative OVCA433 and ER-positive SKOV3 cells. (D) Suppression of p-STAT3 was confirmed by knockdown of STAT3 expression using siRNA. (E) The inhibitory effect of bazedoxifene on the proliferation of OVCA433 and SKOV3 cells after transfection with siNC or siSTAT3-4 is shown $\left({ }^{*} \mathrm{P}<0.05,{ }^{* *} \mathrm{P}<0.01,{ }^{* * *} \mathrm{P}<0.001\right.$; NS, not significant). STAT3, signal transducer and activator of transcription 3 ; NC, negative control.

results showed that treatment with bazedoxifene alone or in combination with paclitaxel induced cell cycle arrest (Fig. 6A). In addition, we demonstrated an apoptotic effect in ovarian cancer cells treated with bazedoxifene and paclitaxel alone or in combination. Paclitaxel, an anticancer drug, is an anthracycline drug that suppresses cancer by inducing apoptosis. In the above experiments, bazedoxifene significantly inhibited the viability of ovarian cancer cell lines. The combination of bazedoxifene and paclitaxel reduced the levels of Mcl-1,
Bcl-xl, and Bcl-2, and significantly increased the levels of Bax in the ovarian cancer OVCA433 and SKOV3 cells (Fig. 6B). The whole western membrane and expression levels are shown in Figs. S15 and S16. Moreover, flow cytometry analysis using Annexin V-fluorescein isothiocyanate (FITC)/propidium iodide (PI) double staining revealed that the combined treatment of bazedoxifene and paclitaxel significantly induced the apoptosis of OVCA433 and SKOV3 cells (Fig. 6C and D). A2780 and TOV112D cells also showed significantly increased 
A

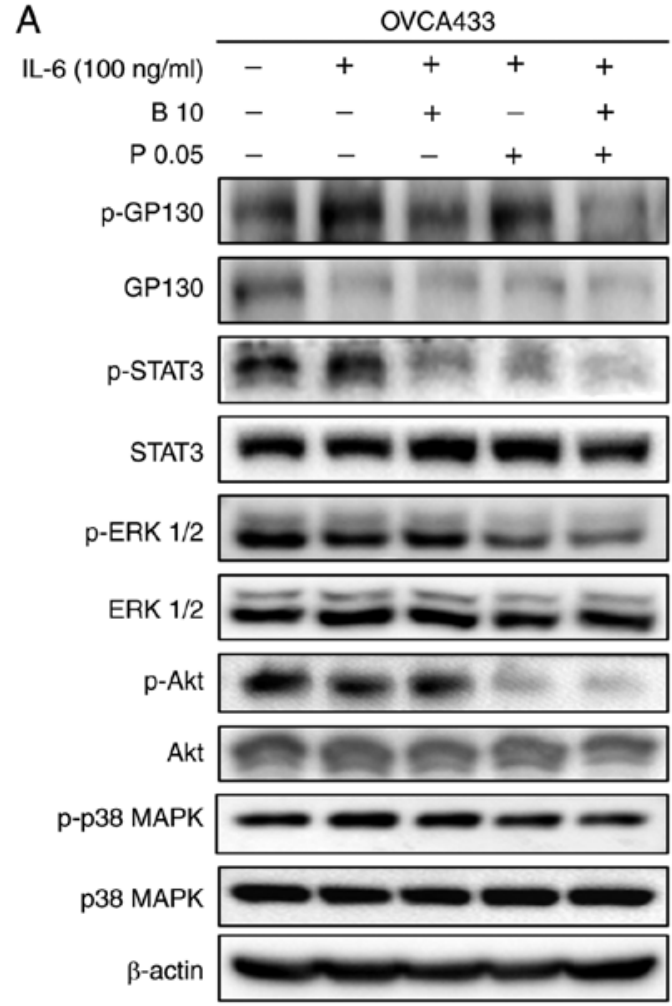

B

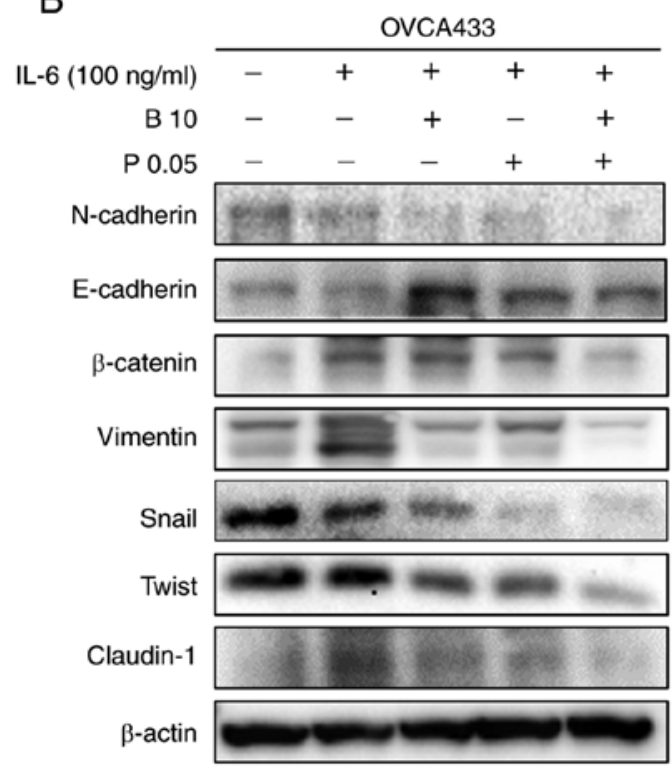

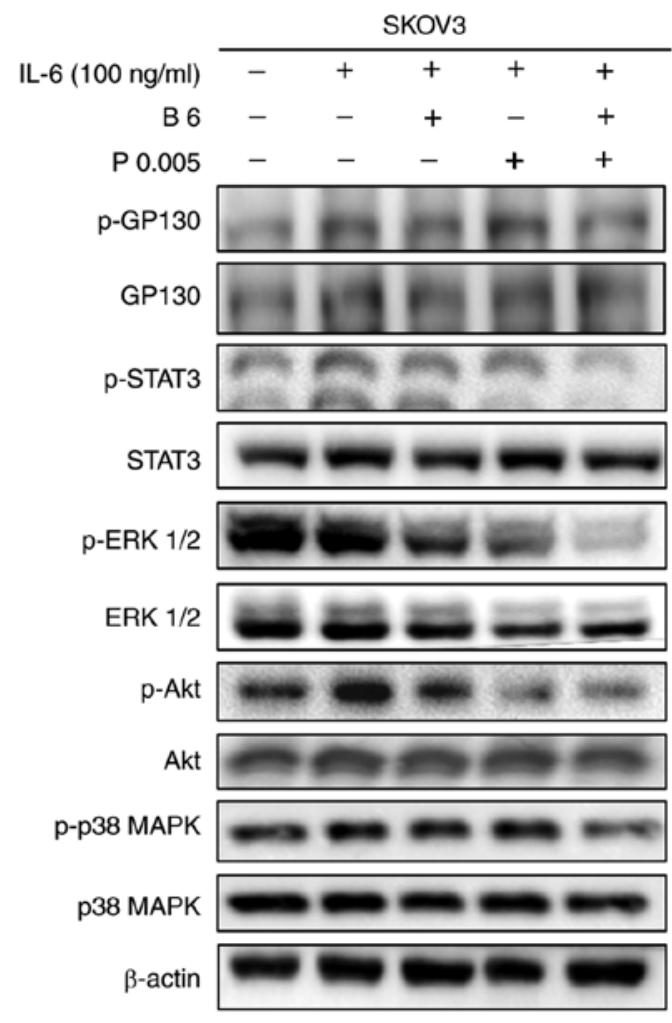

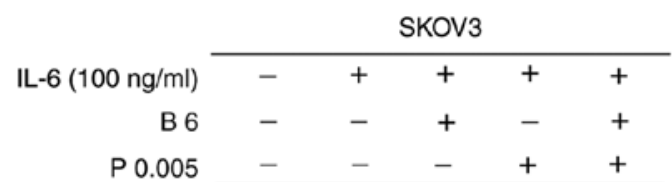

$\mathrm{N}$-cadherin
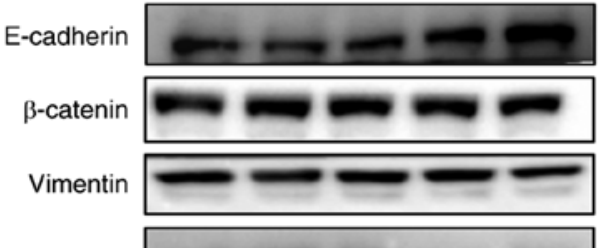

Snail

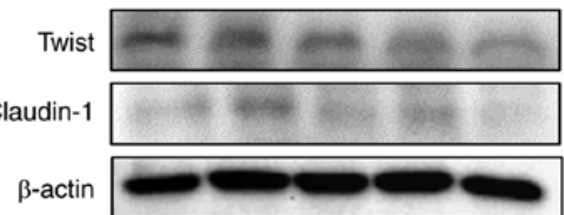

B: Bazedoxifene $(\mu \mathrm{M}), \mathrm{P}:$ Paclitaxel $(\mu \mathrm{g} / \mathrm{ml})$

Figure 5. The combination of bazedoxifene and paclitaxel inhibits the expression of phosphorylated (p)-GP130, p-STAT3, p-ERK1/2 and EMT-related proteins in ovarian cancer cells. (A) Combined bazedoxifene and paclitaxel decreased the expression of p-GP130, p-STAT3 and p-ERK 1/2 in ovarian cancer OVCA433 and SKOV3 cells. (B) Effect of combined treatment with bazedoxifene and paclitaxel on N-cadherin, E-cadherin, $\beta$-catenin, vimentin, Snail, Twist, and Claudin-1 on EMT marker in OVCA433 and SKOV3 cells. EMT, epithelial-mesenchymal transition

apoptosis following treatment with the combination of paclitaxel and bazedofixene (Fig. S17). These results confirmed that both bazedoxifene and paclitaxel induced apoptosis, and apoptosis increased significantly when both drugs were used together.

Combined bazedoxifene and paclitaxel inhibits OVCA433 tumour growth in vivo. OVCA433 cells $\left(1 \times 10^{7}\right)$ were injected subcutaneously into BALB/c nude mice with an equal volume of phosphate-buffered saline (PBS). When tumours reached a volume of $200 \mathrm{~mm}^{3}$, bazedoxifene $(4 \mathrm{mg} / \mathrm{kg})$ was administered with $0.05 \% \mathrm{CMC}$ and $5 \%$ DMSO as a vehicle by gavage five times a week, and paclitaxel $(10 \mathrm{mg} / \mathrm{kg})$ was administered by intraperitoneal injection twice weekly. Tumour volumes were calculated using caliper measurements ( $n=6$ mice per treatment group) (Fig. 7A). After 16 days of treatment, all 
A

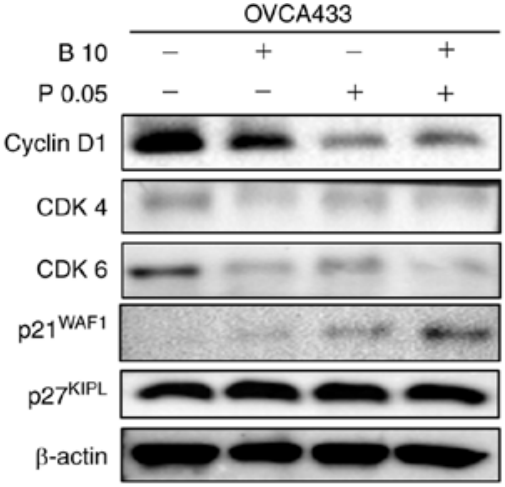

B

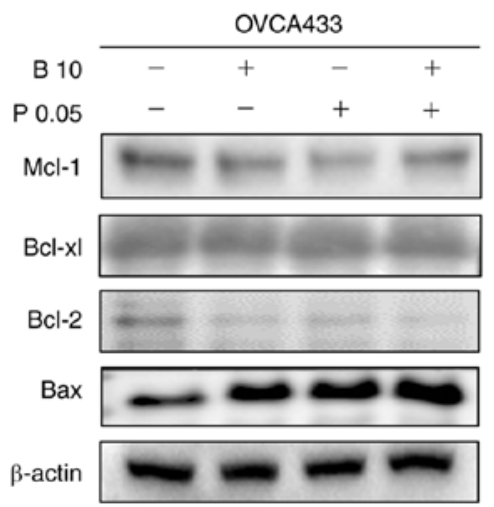

C
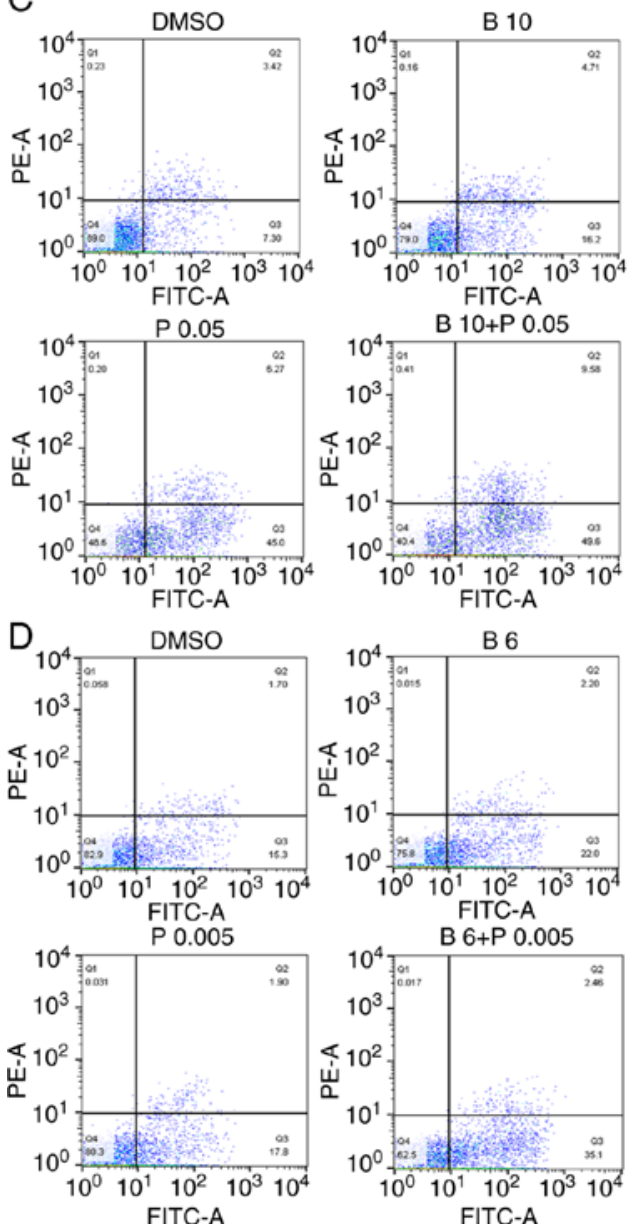

B 6
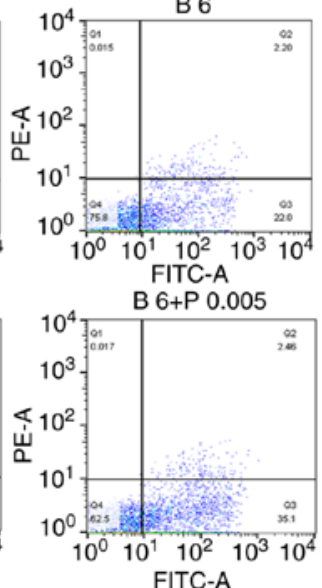
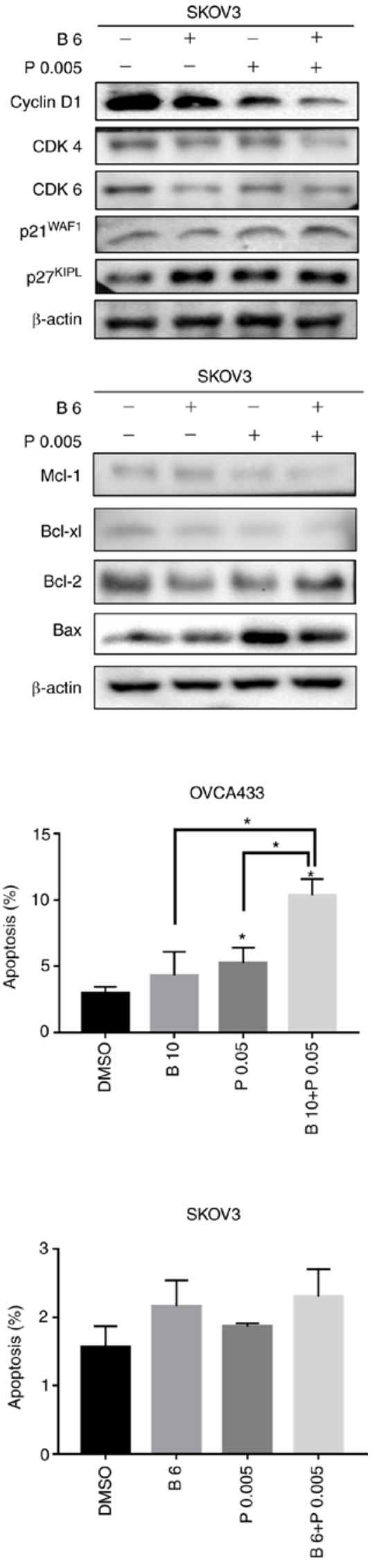

B: Bazedoxifene $(\mu \mathrm{M})$, P: Paclitaxel $(\mu \mathrm{g} / \mathrm{ml})$

Figure 6. Bazedoxifene combined with paclitaxel induce the growth arrest and apoptosis of ovarian cancer cells more significantly than either drug alone. (A) Combined treatment with bazedoxifene and paclitaxel decreased the expression of cyclin D1, CDK4, CDK6, and increased the expression of p21 and p27 in ovarian cancer OVCA433 and SKOV3 cells. (B) Combined bazedoxifene and paclitaxel decreased the expression of Mcl-1, Bcl-xl, Bcl-2, and increased the expression of Bax in OVCA433 and SKOV3 cells. (C and D) OVCA433 and SKOV3 cells were treated with bazedoxifene (B) or/and paclitaxel (P/B+P) in triplicate for $48 \mathrm{~h}$ to detect cell apoptosis using FITC Annexin V apoptosis Kit $(" \mathrm{P}<0.05)$. 
A

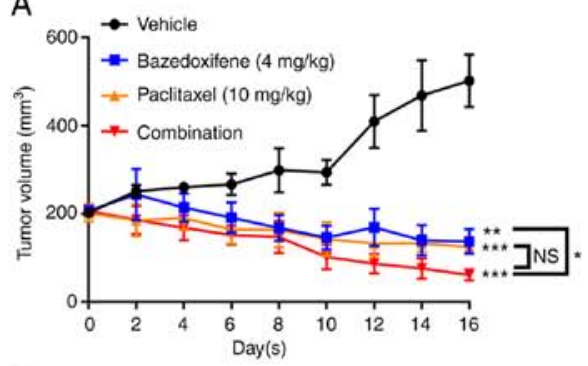

C

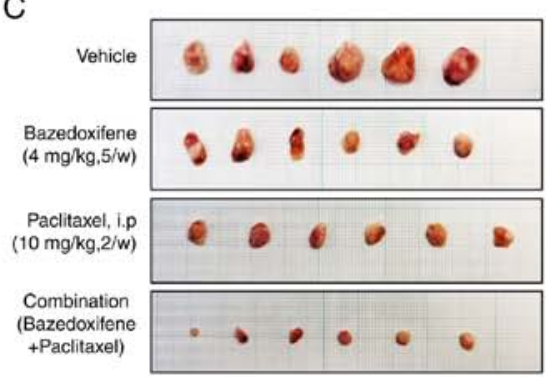

D

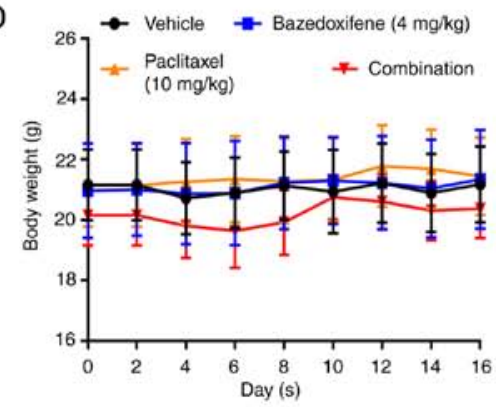

F
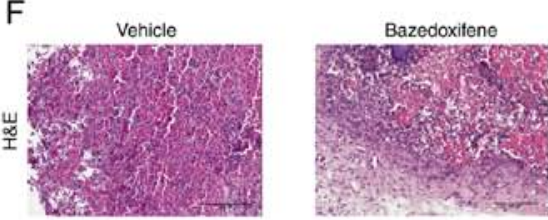

G

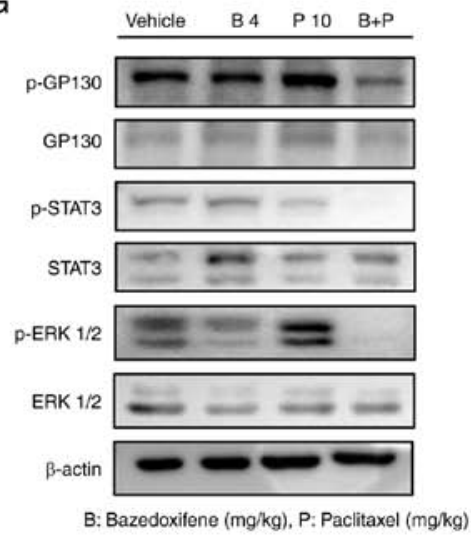

B
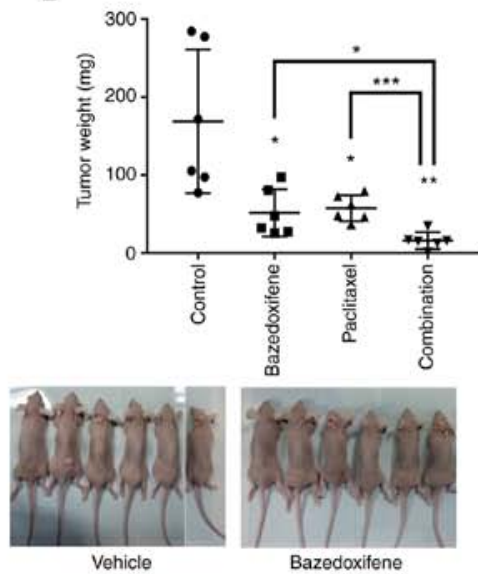

Vehicle

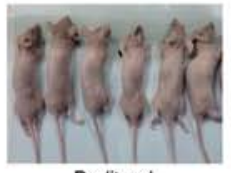

Paclitaxel

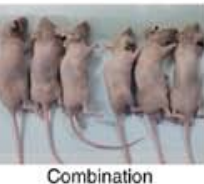

E

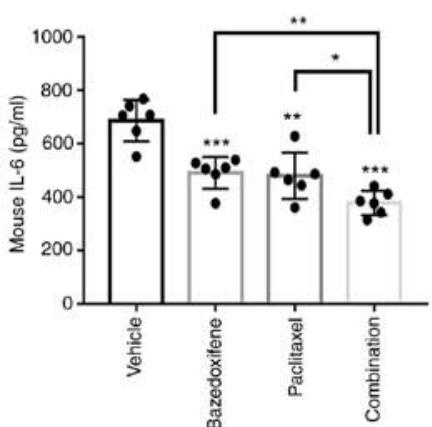

Paclitaxel

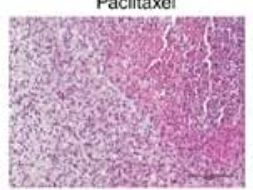

Combination

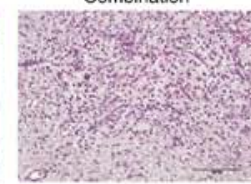

$\mathrm{H}$

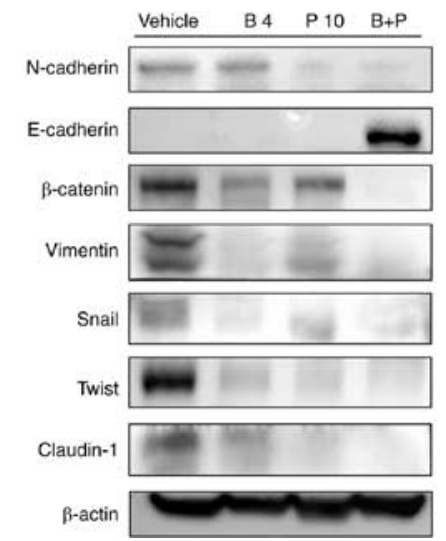

Figure 7. Combined bazedoxifene and paclitaxel inhibits ovarian cancer OVCA433 tumour growth in vivo. OVCA433 cells (1x10 ${ }^{7}$ ) were injected subcutaneously into Balb/c-nude mice with an equal volume of PBS. When tumours reached a volume of $200 \mathrm{~mm}^{3}$, bazedoxifene (4 mg/ $\left.\mathrm{kg}\right)$ with a vehicle of $0.05 \% \mathrm{CMC}$ and 5\% DMSO was administered by gavage five time a week, and paclitaxel $(10 \mathrm{mg} / \mathrm{kg})$ was administered by intraperitoneal (i.p.) injection twice weekly. (A) Tumour volumes were calculated from caliper measurements. (B) After 16 days of treatment, all mice were sacrificed, and the total mass (in mg) of each tumour was determined at autopsy ( $\mathrm{n}=6$ mice per treatment group). (C) The tumour masses were excised for comparison among the groups. (D) The mouse body weights were assessed on the days indicated. (E) To assess IL-6 production per serum in mouse blood, the total amount of IL-6 was normalised by the vehicle in the different treatment groups $\left({ }^{*} \mathrm{P}<0.05,{ }^{* *} \mathrm{P}<0.01,{ }^{* * *} \mathrm{P}<0.001\right)$. (F) Hematoxylin and eosin (H\&E) staining results show the anticancer effect of combined bazedoxifene and paclitaxel on ovarian cancer. (G) The phosphorylation levels (p) of GP130, STAT3 and ERK1/2 were determined using western blotting of the harvested tumour tissue. $\beta$-actin served as the loading control. $(\mathrm{H})$ The protein levels of N-cadherin, E-cadherin, $\beta$-catenin, Vimentin, Snail, Twist and Claudin-1 were determined using western blotting of the harvested tumour tissue. $\beta$-actin served as the loading control. STAT3, signal transducer and activator of transcription 3; GP130, glycoprotein 130. 
mice were sacrificed, and the total mass of each tumour was determined at autopsy. The tumour mass was excised for comparison between groups (Fig. 7B and C). There was no change in the body weight of the mice during the drug treatment period (Fig. 7D). To assess IL-6 production per serum in mouse blood, the total amount of IL- 6 was normalised to that of the vehicle (Fig. 7E). Histological examination revealed fewer cells with large nucleoli and irregular nuclear membranes in the drug-treated group than in the vehicle group, especially in the drug combination group (Fig. 7F). The phosphorylation levels of GP130, STAT3, and ERK were determined by western blotting of the harvested tumour tissue. $\beta$-actin served as a loading control. The protein levels of $\mathrm{N}$-cadherin, E-cadherin, $\beta$-catenin, vimentin, Snail, Slug and Claudin-1 were determined by western blotting of the harvested tumour tissue. $\beta$-actin served as a loading control (Fig. $7 \mathrm{G}$ and $\mathrm{H}$ ). The whole western membrane and expression levels are shown in Figs. S18 and S19. The results of experiments using a xenograft mouse model showed that the combination treatment of bazedoxifene and paclitaxel significantly reduced the tumour volume and weight. In addition, GP130/STAT3 signalling and EMT signalling were inhibited by the combination treatment in mouse tumour tissues. This supports the results of the in vitro experiments.

\section{Discussion}

Interleukin-6 (IL-6) plays several roles in haematopoiesis and regeneration. In addition, its function plays a central role in cancer progression and development. The expression of IL-6 is upregulated in several malignancies, and enhanced IL-6 signalling increases IL-6 expression by inducing sustained activation of the transcription factor signal transducer and activator of transcription 3 (STAT3). Therefore, it provides a tumour microenvironment that promotes tumour growth (27). IL-6 is known to be involved in cancer progression and resistance in a variety of cancers and is involved in sub-STAT3 signalling $(28,29)$. IL-6 signalling is induced by the binding of IL-6 to IL-6R. IL-6, which is complexed with IL-6R, binds to glycoprotein 130 (GP130), known as IL-6R, with high affinity and activates the homodimer (30). Because GP130 is located in the middle of this oncogenic signalling network, blocking GP130 can be an important treatment method for suppressing carcinogenesis and tolerance. However, several drugs that inhibit GP130 have not been developed, and studies on cancer have not been conducted. Currently, SC144 is an inhibitor of GP130 in ovarian cancer, and studies have been conducted on cancer inhibition and resistance (31).

Bazedoxifene, a GP130 inhibitor, has been used as a selective estrogen modulator (SERM) drug to prevent and treat osteoporosis in women after menopause. Recently, it was discovered that it selectively inhibits IL-6-induced STAT3 phosphorylation in the GP130/JAK/STAT3 signalling pathway in cancer (18). Using surface plasmon resonance (SPR), we confirmed that bazedoxifene binds to GP130 through intermolecular interactions. To confirm that binding to GP130 inhibited the binding of IL-6, we examined whether IL-6 was inhibited by the IL-6-dependent cell line DS-1. The results showed that IL-6 inhibition increased with increasing concentrations of bazedoxifene.
We aimed to determine whether bazedoxifene selectively inhibits IL-6-induced STAT3 phosphorylation through the GP130/JAK/STAT3 signalling pathway in ovarian cancer. IL-6-mediated STAT3 phosphorylation in A2780 and SKOV3 cells, where STAT3 phosphorylation is absent in ovarian cancer, was inhibited by bazedoxifene. In addition, it was confirmed that STAT3 phosphorylation was inhibited through inhibition of the STAT3 signalling pathway even in the absence of estrogen receptors (ERs), which are the targets of bazedoxifene. This demonstrates that bazedoxifene inhibits the IL-6/GP130/STAT3 signalling pathway with and without ERs in ovarian cancer.

To confirm the effect of bazedoxifene on ovarian cancer cell lines, the cells were treated with bazedoxifene at each concentration. The survival of ovarian cancer cells was inhibited by increasing the concentration of bazedoxifene. Thus, bazedoxifene could be used to inhibit the growth of ovarian cancer cells, and the combined effect of bazedoxifene and paclitaxel could be used as an anticancer agent. Subsequently, we investigated the binding effect of bazedoxifene and paclitaxel on the migration and invasion of cancer cells. Both migration and invasion showed decreased results compared with the control group, even with single treatment, and decreased further with the combination treatment. The results showed that bazedoxifene suppressed survival, migration, and invasion by a combination treatment with paclitaxel.

Survival, migration, and invasion were inhibited by bazedoxifene-mediated inhibition of GP130 through the STAT3 signalling system. The results demonstrated that the phosphorylation of GP130, STAT3, and ERK1/2 was inhibited in combination with bazedoxifene, paclitaxel, and bazedoxifene and paclitaxel. STAT3 is continuously activated in several types of cancers, and is associated with the incidence and progression of cancer, as well as the prognosis of cancer patients $(28,32)$. Constitutive STAT3 activation not only has intrinsic consequences for tumour cells but also affects the extracellular matrix (ECM) of the tumour microenvironment and stromal cells, thereby increasing the survival, proliferation, migration, and invasiveness of tumour cells and their tumor-promoting activity (33). Thus, bazedoxifene inhibited IL-6-induced phosphorylation of STAT3 and inhibited the expression of genes downstream of IL-6. Because it could affect the tumour microenvironment, the effect on EMT in the tumour microenvironment was confirmed. In the EMT signalling pathway, bazedoxifene and paclitaxel inhibited EMT, and the combination treatment showed greater inhibition.

We demonstrated that bazedoxifene inhibits the survival, migration, and metastasis of cancer cells by inhibiting IL-6/GP130/STAT3 signalling. STAT3 plays a central role in Gp130-mediated cell growth, survival, and differentiation. STAT3 is activated by various oncogenes and is involved in cell growth and differentiation through $\mathrm{G} 1$ to $\mathrm{S}$ phase cell cycle regulation (34). Apoptosis is an essential sign of cell death and is associated with the development of several tumours (35). Paclitaxel, an anticancer drug, induces apoptosis by stabilising microtubule dynamics (36-38). In addition, constitutive activation of STAT3 induces resistance to apoptosis, presumably by upregulating the expression of survivin, Bcl-xl, and Bcl-2 (39). We investigated whether bazedoxifene affected cell growth and differentiation through GP130/STAT3 inhibition. Thus, it 
was confirmed that the drug alone or combination treatment inhibited the growth and differentiation of cells. We found that cyclin D1, CDK4, and CDK6 decreased significantly, and p21 and p27 increased slightly in the cell treated with bazedoxifene and paclitaxel alone and in the combination group. We also investigated the apoptotic effects of bazedoxifene and paclitaxel. In the above experiment, bazedoxifene significantly inhibited the viability of ovarian cancer cell lines. These results confirmed that both bazedoxifene and paclitaxel induced apoptosis, and apoptosis significantly increased when both drugs were used together.

In addition, we found that bazedoxifene inhibited the growth of transplanted tumours in immunosuppressed mice, improved the sensitivity of traditional anticancer drugs, and exerted synergistic effects. The expression of STAT3 signalling and EMT was more inhibited in the group treated with the combination of bazedoxifene and paclitaxel as compared to the single treatment group.

In this study, we investigated the effect of bazedoxifene on cancer cell growth and metastasis by inhibiting IL-6/GP130/STAT3 signalling in ovarian cancer cells. Furthermore, we investigated the effect of bazedoxifene alone or in combination with paclitaxel on the growth and metastasis inhibition of ovarian cancer. Bazedoxifene increased the sensitivity of ovarian cancer cells to paclitaxel. The antitumour effect of the combination of bazedoxifene and paclitaxel significantly improved the therapeutic efficacy of ovarian cancer. This suggests its potential as an adjuvant for anticancer drugs.

In conclusion, in this study, bazedoxifene inhibited IL-6 by binding to GP130. Bazedoxifene inhibited cell survival in ovarian cancer cells, and combination therapy with bazedoxifene and paclitaxel further inhibited cell survival, migration, and invasion compared to treatment with either drug alone. In addition, combination treatment with bazedoxifene and paclitaxel inhibited the IL-6-mediated GP130/STAT3 signalling pathway, induced apoptosis in ovarian cancer cells, and inhibited EMT. Tumour growth was suppressed in human ovarian cancer xenografts. These results suggest that bazedoxifene can be a novel therapeutic agent for ovarian cancer treatment, and can be used as an adjunct to the existing anticancer drug, paclitaxel.

\section{Acknowledgements}

Not applicable.

\section{Funding}

This work was supported by the Basic Science Research Program through the National Research Foundation of Korea (NRF), funded by the Ministry of Education, Science, and Technology (grant nos. NRF-2018R1D1A1B07 049780, 2018R1A6A1A03025108, 2021R1A2C2009782 and 2021R1A6A3A1303840811).

\section{Availability of data and materials}

The datasets used during the present study are available from the corresponding author upon reasonable request.

\section{Authors' contributions}

SAP was responsible for the data curation, writing of the original draft, visualization, investigation, software, validation of the data, writing of the review, and editing. LKK was responsible for the data curation, methodology, and validation of the data and results. HMP was responsible for the research methodology. HJK was responsible for the data curation, conceptualization, methodology, software, supervision, project administration, writing of the review, and editing. THH was responsible for the conceptualization, data curation and validation, visualization, supervision, project administration, and funding acquisition. All authors have read and agreed to the published version of the manuscript.

\section{Ethics approval and consent to participate}

Animal experiments were approved by the Institutional Animal Care and Use Committee (IACUC) of the Catholic University of Korea (approval no. CUK-IACUC-2019-026-01). All experimental work complied with the legal obligations and federal guidelines for the care and maintenance of laboratory animals.

\section{Patient consent for publication}

Not applicable.

\section{Competing interests}

The authors declare that there are no competing interests for any of the authors.

\section{References}

1. Siegel RL, Miller KD and Jemal A: Cancer statistics, 2018. CA Cancer J Clin 68: 7-30, 2018.

2. Markman M and Mekhail TM: Paclitaxel in cancer therapy. Expert Opin Pharmacother 3: 755-766, 2002.

3. Richardson DL, Sill MW, Coleman RL, Sood AK, Pearl ML, Kehoe SM, Carney ME, Hanjani P, Van Le L, Zhou XC, et al: Paclitaxel with and without pazopanib for persistent or recurrent ovarian cancer: A randomized clinical trial. JAMA Oncol 4: 196-202, 2018.

4. Pignata S, Lorusso D, Scambia G, Sambataro D, Tamberi S, Cinieri S, Mosconi AM, Orditura M, Brandes AA, Arcangeli V, et al: Pazopanib plus weekly paclitaxel versus weekly paclitaxel alone for platinum-resistant or platinum-refractory advanced ovarian cancer (MITO 11): A randomised, open-label, phase 2 trial. Lancet Oncol 16: 561-568, 2015.

5. Browning L, Patel M, Bring Horvath E, Tawara K and Jorcyk C: IL-6 and ovarian cancer: Inflammatory cytokines in promotion of metastasis. Cancer Manag Res 10: 6685-6693, 2018.

6. Heo TH, Wahler J and Suh N: Potential therapeutic implications of IL-6/IL-6R/gp130-targeting agents in breast cancer. Oncotarget 7: 15460, 2016.

7. Ernst $\mathrm{M}$ and Jenkins BJ: Acquiring signalling specificity from the cytokine receptor gp130. Trends Genet 20: 23-32, 2004.

8. Rebouissou S, Amessou M, Couchy G, Poussin K, Imbeaud S, Pilati C, Izard T, Balabaud C, Bioulac-Sage P and Zucman-Rossi J: Frequent in-frame somatic deletions activate gp130 in inflammatory hepatocellular tumours. Nature 457: 200-204, 2009.

9. Murakami M, Kamimura D and Hirano T: Pleiotropy and specificity: Insights from the interleukin 6 family of cytokines. Immunity 50: 812-831, 2019.

10. Ernst M and Putoczki TL: Molecular pathways: IL11 as a tumor-promoting cytokine-translational implications for cancers. Clin Cancer Res 20: 5579-5588, 2014. 
11. Rose-John S: Interleukin-6 family cytokines. Cold Spring Harb Perspect Biol 10: a028415, 2018.

12. Bromberg J and Wang TC: Inflammation and cancer: IL-6 and STAT3 complete the link. Cancer Cell 15: 79-80, 2009.

13. Haan C, Heinrich PC and Behrmann I: Structural requirements of the interleukin- 6 signal transducer gp130 for its interaction with Janus kinase 1: The receptor is crucial for kinase activation. Biochem J 361: 105-111, 2002.

14. Taher MY, Davies DM and Maher J: The role of the interleukin (IL)-6/IL-6 receptor axis in cancer. Biochem Soc Trans 46: $1449-1462,2018$

15. Skiniotis G, Boulanger MJ, Garcia KC and Walz T: Signaling conformations of the tall cytokine receptor gp130 when in complex with IL-6 and IL-6 receptor. Nat Struct Mol Biol 12: 545-551, 2005.

16. Dijkgraaf EM, Welters MJ, Nortier JW, van der Burg SH and Kroep JR: Interleukin-6/interleukin-6 receptor pathway as a new therapy target in epithelial ovarian cancer. Curr Pharm Des 18: 3816-3827, 2012

17. Levy DE and Darnell JE Jr: Stats: Transcriptional control and biological impact. Nat Rev Mol Cell Biol 3: 651-662, 2002.

18. Li H, Xiao H, Lin L, Jou D, Kumari V, Lin J and Li C: Drug design targeting protein-protein interactions (PPIs) using multiple ligand simultaneous docking (MLSD) and drug repositioning: Discovery of raloxifene and bazedoxifene as novel inhibitors of IL-6/GP130 interface. J Med Chem 57: 632-641, 2014.

19. Fu S, Chen X, Lo HW and Lin J: Combined bazedoxifene and paclitaxel treatments inhibit cell viability, cell migration, colony formation, and tumor growth and induce apoptosis in breast cancer. Cancer Lett 448: 11-19, 2019.

20. Kim L, Park S, Park H, Kim H and Heo TH: Bazedoxifene, a GP130 inhibitor, modulates EMT signaling and exhibits antitumor effects in HPV-positive cervical cancer. Int J Mol Sci 22: 8693,2021

21. Wu X, Cao Y, Xiao H, Li C and Lin J: Bazedoxifene as a novel GP130 inhibitor for pancreatic cancer therapy. Mol Cancer Ther 15: 2609-2619, 2016.

22. Tian J, Chen X, Fu S, Zhang R, Pan L, Cao Y, Wu X, Xiao H, Lin HJ, Lo HW, et al: Bazedoxifene is a novel IL-6/GP130 inhibitor for treating triple-negative breast cancer. Breast Cancer Res Treat 175: 553-566, 2019.

23. Wei J, Ma L, Lai YH, Zhang R, Li H, Li C and Lin J: Correction to: Bazedoxifene as a novel GP130 inhibitor for colon cancer therapy. J Exp Clin Cancer Res 38: 374, 2019.

24. Hong SS, Choi JH, Lee SY, Park YH, Park KY, Lee JY, Kim J, Gajulapati V, Goo JI, Singh S, et al: A novel small-molecule inhibitor targeting the IL- 6 receptor $\beta$ subunit, glycoprotein 130 . J Immunol 195: 237-245, 2015.

25. Beaufort CM, Helmijr JC, Piskorz AM, Hoogstraat M, Ruigrok-Ritstier K, Besselink N, Murtaza M, van IJcken WF, Heine AA, Smid M, et al: Ovarian cancer cell line panel (OCCP): Clinical importance of in vitro morphological subtypes. PLoS One 9: e103988, 2014
26. Fu W, Zhao P, Li H, Fu H, Liu X, Liu Y, Wu J and Fu W: Bazedoxifene enhances paclitaxel efficacy to suppress glioblastoma via altering Hippo/YAP pathway. J Cancer 11: 657-667, 2020.

27. Chang Q, Daly L and Bromberg J: The IL-6 feed-forward loop: A driver of tumorigenesis. Semin Immunol 26: 48-53, 2014.

28. Huang M, Page C, Reynolds RK and Lin J: Constitutive activation of stat 3 oncogene product in human ovarian carcinoma cells. Gynecol Oncol 79: 67-73, 2000.

29. Duan Z, Ames RY, Ryan M, Hornicek FJ, Mankin H and Seiden MV: CDDO-Me, a synthetic triterpenoid, inhibits expression of IL-6 and Stat 3 phosphorylation in multi-drug resistant ovarian cancer cells. Cancer Chemother Pharmacol 63: 681-689, 2009.

30. Wolf J, Rose-John S and Garbers C: Interleukin-6 and its receptors: A highly regulated and dynamic system. Cytokine 70 11-20, 2014.

31. Xu S, Grande F, Garofalo A and Neamati N: Discovery of a novel orally active small-molecule gp130 inhibitor for the treatment of ovarian cancer. Mol Cancer Ther 12: 937-949, 2013.

32. Huang F, Tong X, Fu L and Zhang R: Knockdown of STAT3 by shRNA inhibits the growth of CAOV3 ovarian cancer cell line in vitro and in vivo. Acta Biochim Biophys Sin (Shanghai) 40: 519-525, 2008

33. Yu H, Kortylewski M and Pardoll D: Crosstalk between cancer and immune cells: Role of STAT3 in the tumour microenvironment. Nat Rev Immunol 7: 41-51, 2007.

34. Hirano T, Ishihara K and Hibi M: Roles of STAT3 in mediating the cell growth, differentiation and survival signals relayed through the IL-6 family of cytokine receptors. Oncogene 19: 2548-2556, 2000.

35. Evan GI and Vousden KH: Proliferation, cell cycle and apoptosis in cancer. Nature 411: 342-348, 2001.

36. Ling YH, Yang Y, Tornos C, Singh B and Perez-Soler R: Paclitaxel-induced apoptosis is associated with expression and activation of c-Mos gene product in human ovarian carcinoma SKOV3 cells. Cancer Res 58: 3633-3640, 1998.

37. Orr GA, Verdier-Pinard P, McDaid $\mathrm{H}$ and Horwitz SB: Mechanisms of taxol resistance related to microtubules. Oncogene 22: 7280-7295, 2003.

38. Jones NA, Turner J, McIlwrath AJ, Brown R and Dive C: Cisplatin-and paclitaxel-induced apoptosis of ovarian carcinoma cells and the relationship between bax and bak up-regulation and the functional status of p53. Mol Pharmacol 53: 819-826, 1998.

39. Nielsen M, Kaestel CG, Eriksen K, Woetmann A, Stokkedal T, Kaltoft K, Geisler C, Röpke C and Odum N: Inhibition of constitutively activated Stat 3 correlates with altered Bcl-2/Bax expression and induction of apoptosis in mycosis fungoides tumor cells. Leukemia 13: 735-738, 1999.

(i) $($ This work is licensed under a Creative Commons Attribution-NonCommercial-NoDerivatives 4.0 International (CC BY-NC-ND 4.0) License. 